\title{
A comparison of control samples for ChIP-seq of histone modifications
}

\author{
Christoffer Flensburg $^{1 \dagger}$, Sarah A. Kinkel ${ }^{2}$, Andrew Keniry ${ }^{2}$, Marnie E. Blewitt $^{2,3}$ and Alicia Oshlack ${ }^{1,3 *}$ \\ 1 Bioinformatics Group, Murdoch Childrens Research Institute, Melbourne, VIC, Australia \\ ${ }^{2}$ Division of Molecular Medicine, Department of Medical Biology, Walter and Eliza Hall Institute, University of Melbourne, Melbourne, VIC, Australia \\ ${ }^{3}$ Department of Genetics, University of Melbourne, Melbourne, VIC, Australia
}

\section{Edited by:}

Mattia Pelizzola, Istituto Italiano di

Tecnologia, Italy

Reviewed by:

Raffaele A. Calogero, University of Torino, Italy

Thiruvarangan Ramaraj, National

Center for Genome Resources, USA

\section{*Correspondence:}

Alicia Oshlack, Bioinformatics

Group, Murdoch Childrens Research Institute, Royal Children's Hospital,

Parkville, VIC 3052, Australia

e-mail: alicia.oshlack@mcri.edu.au

${ }^{\dagger}$ Present address:

Christoffer Flensburg, Cancer and Haematology Division, Department of Medical Biology, Walter and Eliza Hall Institute, University of Melbourne, Parkville, Australia
The advent of high-throughput sequencing has allowed genome wide profiling of histone modifications by Chromatin ImmunoPrecipitation (ChIP) followed by sequencing (ChIP-seq). In this assay the histone mark of interest is enriched through a chromatin pull-down assay using an antibody for the mark. Due to imperfect antibodies and other factors, many of the sequenced fragments do not originate from the histone mark of interest, and are referred to as background reads. Background reads are not uniformly distributed and therefore control samples are usually used to estimate the background distribution at any given genomic position. The Encyclopedia of DNA Elements (ENCODE) Consortium guidelines suggest sequencing a whole cell extract (WCE, or "input") sample, or a mock ChIP reaction such as an IgG control, as a background sample. However, for a histone modification ChIP-seq investigation it is also possible to use a Histone $\mathrm{H} 3(\mathrm{H} 3)$ pull-down to map the underlying distribution of histones. In this paper we generated data from a hematopoietic stem and progenitor cell population isolated from mouse fetal liver to compare WCE and H3 ChIP-seq as control samples. The quality of the control samples is estimated by a comparison to pull-downs of histone modifications and to expression data. We find minor differences between WCE and H3 ChIP-seq, such as coverage in mitochondria and behavior close to transcription start sites. Where the two controls differ, the $\mathrm{H} 3$ pull-down is generally more similar to the ChIP-seq of histone modifications. However, the differences between $\mathrm{H} 3$ and WCE have a negligible impact on the quality of a standard analysis.

Keywords: ChIP-seq, histone modifications, control sample, whole cell extract, input, H3, quality control

\section{INTRODUCTION}

As sequencing grows cheaper and more efficient (Koboldt et al., 2013), ChIP-seq of histone modifications is becoming an increasingly powerful tool to understand the underlying mechanics of gene regulation (Trynka et al., 2013; Zhu et al., 2013). Studies utilizing ChIP-seq of histone modifications are now common in research, and large public data sets are also available through consortia such as Encyclopedia of DNA Elements (ENCODE), (Consortium, 2004) or Epigenomics Roadmap (Bernstein et al., 2010). The ChIP protocol is complicated however, and it is often hard to achieve consistently high quality data (Park, 2009). A necessity of the ChIP protocol is the use of antibodies, which inherently have varying specificity (Bock et al., 2011) that can cause fragments unrelated to the desired target to be sequenced. Furthermore, there are biases in the sequencing process and alignment, such as PCR amplification artifacts, GC biases and alignment artifacts. See Park (2009) for further discussion of error sources in ChIP-seq.

A widely accepted technique for handling these biases is the use of control samples that exhibit similar biases as the ChIP samples, but without the enrichment related to the ChIP target. The enrichment of the target histone modification can then be extracted by comparing the ChIP to the control samples. This procedure can eliminate a wide range of error sources, but hinges on the quality and analysis of the control as much as on the ChIP (Ho et al., 2011; Landt et al., 2012; Liang and Keles, 2012).

The most common control samples used are whole cell extract (WCE) or a mock pull down. The WCE is a sample of the sheared chromatin taken prior to immunoprecipitation and is often referred to as the "input," whereas a mock pull down gives an estimation of background by pulling down an irrelevant target using a non-specific antibody such as IgG. Both of these options emulate some of the biases present in a ChIP-seq data set but a mock pull-down is believed to be more similar to the background signal of the ChIP sample as it emulates more steps in the processing of the sample. However, it can be difficult to retrieve sufficient amounts of DNA from a mock immunoprecipitation to give an accurate estimation of the background and so a WCE sample is by far the most common control sample used (Kidder et al., 2011; Landt et al., 2012).

An alternative control for the ChIP-seq of Histone H3 modifications is an anti-H3 antibody immunoprecipitation, which closely mimics the background by enriching the sample at the location of histones (nucleosomes) along the DNA. Therefore, 
using an $\mathrm{H} 3$ pull-down as a control sample gives a measure of the enrichment in relation to the presence of a histone. For example, if a histone modification antibody had a slight affinity for all histones whether or not they had the modification, the $\mathrm{H} 3$ pulldown could account for this background. This is slightly different to a WCE control that not only misses the immunoprecipitation process in the protocol but also attempts to measure the density of a modified histone relative to a uniform genome. Here we investigate which background sample is the most efficient at reducing noise and extracting biologically relevant information from histone modification ChIP-seq data.

To our knowledge there are no studies comparing WCE or IgG to Histone H3 immunoprecipitation as a control sample, and there are few studies using $\mathrm{H} 3$ immunoprecipitation as a control. In this paper we use new unpublished data from a mouse hematopoietic stem and progenitor cell population that includes WCE, H3, and trimethylation of lysine 27 on histone H3 (H3K27me3) ChIP-seq samples, together with RNAseq expression data, to study the differences between WCE and H3 pull down as control samples. This data set shows that H3 samples share some features with the H3K27me3 samples that are not present in the WCE sample, but that these biases do not have a significant impact in most standard analyses.

The first part of this paper studies the genome wide properties of the WCE and H3 samples and looks for differences and similarities between the two types of background samples. In the results section we firstly compare each background sample to histone modification ChIP-seq and expression data. We then compare which control is most successful in extracting correlation between histone modifications and expression.

\section{MATERIALS AND METHODS ChIP AND CELL ISOLATION PROTOCOLS}

The mouse hematopoietic stem and progenitor cell population for ChIP was isolated from E14.5 fetal livers from C57BL/6 mice by fluorescence-activated cell sorting based on the following cell surface marker profile: lineage (Ter119, B220, CD5, $\mathrm{CD} 3, \mathrm{Gr} 1)$ negative, $\mathrm{c}^{-\mathrm{Kit}^{+}}$and $\mathrm{Sca}^{+}{ }^{+}$. Similarly, adult bone marrow hematopoietic stem and progenitor cells for RNA-seq were sorted based on lineage (Ter119, B220, CD19, Mac1, Gr1, $\mathrm{CD} 2, \mathrm{CD} 3, \mathrm{CD} 8)$ negative, $\mathrm{c}-\mathrm{Kit}^{+}$and $\mathrm{Sca}^{+}{ }^{+}$cell surface marker expression. Approximately 250,000 cells were used for each ChIP, whereas 30,000-100,000 cells were used to prepare RNA for the RNA-seq.

For chromatin immunoprecipitation (ChIP), formaldehyde cross-linked cells were sonicated in a Covaris sonicator. A small fraction of sonicated material was retained as the WCE sample and the remainder was incubated with either an antibody against $\mathrm{H} 3$ (AbCam) or $\mathrm{H} 3 \mathrm{~K} 27 \mathrm{me} 3$ (Millipore) overnight at $4^{\circ} \mathrm{C}$. Immune complexes were purified by incubation with protein $\mathrm{G}$ beads (Life Technologies) at $4^{\circ} \mathrm{C}$ for an hour. Cross-links were reversed by incubation at $65^{\circ} \mathrm{C}$ for $4 \mathrm{~h}$ before DNA fragments were purified with the ChIP Clean and Concentrator kit (Zymo). Sequencing libraries were prepared using the TruSeq DNA Sample Prep Kit (Illumina) and sequencing was performed on a HiSeq2000 (Illumina).

\section{DATA}

ChIP-seq data was generated for the histone mark H3K27me3 in a hematopoietic stem and progenitor cell population isolated from E14.5 mouse fetal liver from C57BL/6 mice. There are three replicates of $\mathrm{H} 3 \mathrm{~K} 27 \mathrm{me} 3 \mathrm{ChIP}$-seq, with approximately 16, 17, and $18 \mathrm{M}$ reads each, two $\mathrm{H} 3 \mathrm{ChIP}$-seq replicates with approximately 24 and $27 \mathrm{M}$ reads each, and one WCE with $44 \mathrm{M}$ reads. We also generated three RNA-seq replicates from a hematopoietic stem and progenitor cell population isolated from the bone marrow of adult mice that had received fetal liver cell transplants with approximately $17 \mathrm{M}$ reads each. The reads are $100 \mathrm{bp}$ single end reads sequenced by Illumina HiSeq technology. Data is available from GEO (GSE59419).

We used default Bowtie 2 version 2.2.3 with the --verysensitive-local preset and all other settings as default to align the ChIP-seq and WCE reads where data should map directly to the genome. To properly handle RNA fragments that span exon junctions, alignment for the RNA-seq data was performed using TopHat version 2.0.8 with the --b2-very-sensitive preset and all other setting with default parameters. Alignment was performed against mm10 (downloadable from http://hgdownload.soe.ucsc. edu/goldenPath/mm10/bigZips/chromFa.tar.gz).

\section{METHODS}

The aligned reads were filtered for mapping quality of 20 or more, and assigned to 100 and $1000 \mathrm{bp}$ consecutive, non-overlapping bins over the genome based on the center of the read. The smaller bin size is used in the gene coverage analysis to give a sufficient resolution compared to a typical fragment size, while the larger bin size is used for count distribution and differential enrichment analysis, trading some resolution for more statistical power. For some analyses the larger library sizes are downsampled to match the smallest. We want to keep a fraction $p_{i}=N_{\min } / N_{i}$ of the reads from each library $i$, where $N_{i}$ is the library size of library $i$, and $N_{\text {min }}$ is the smallest library size in the comparison. This is implemented by reassigning a bin with $b$ reads in library $i$, a new read count $b^{\prime}$, randomly selected from a binomial distribution $B\left(b, p_{i}\right)$ of $b$ tries with an expectation value of $p_{i} b$.

For comparison purposes, we generated random counts based on a Poisson distribution (Figure 1). Specifically, bins that have no reads in any sample are not assigned any reads in the random sample. The remaining $B$ bins are assigned a random number of reads from a Poisson distribution with an expectation value of $\lambda=N_{\min } / B$.

The MA plots are generated using the plotMA function in the limma R package (Smyth, 2004), where the log fold change is plotted against the mean log intensity.

Differential analysis of counts between the control samples was done with limma-voom (Smyth, 2004; Law et al., 2014), with the replicated histone modification counts used in the variance estimates for each bin.

Peak finding was performed using MACS 2.0.10 (Zhang et al., 2008), with default parameters. Peaks from different samples were classified as overlapping if the peak regions shared at least one base pair (bp).

Expression levels were determined from read counts per million reads per kilobasepair (kb) of exon length (RPKM). The read 


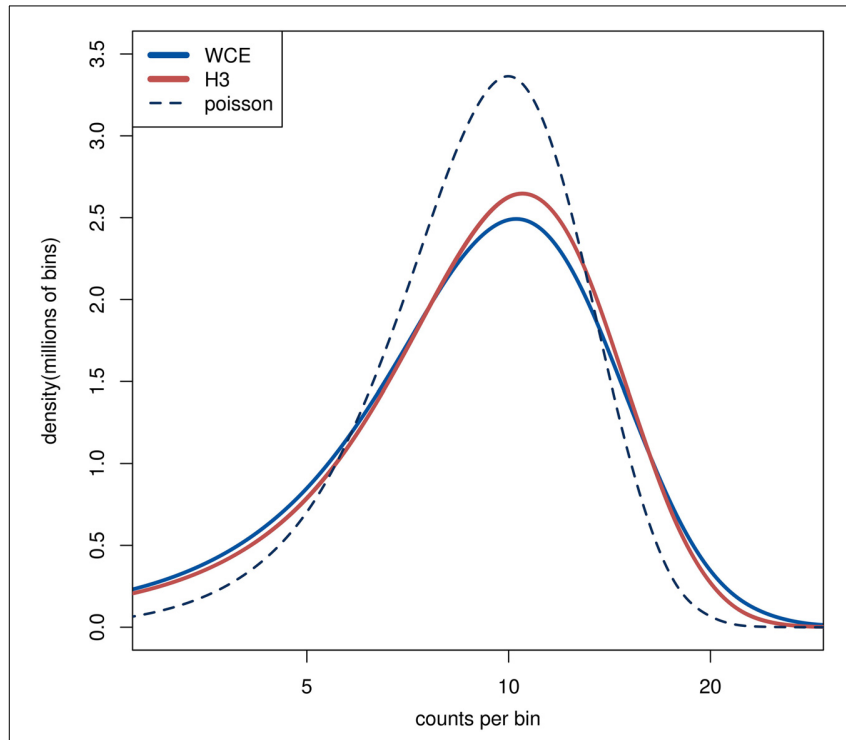

FIGURE 1 | The distribution of read counts in $\mathbf{1}$ kb bins over the genome. The lines from the two $\mathrm{H} 3$ samples are completely overlapping. The dashed line is from a random sample where the reads are distributed independently with equal probability for each bin. The curves are smoothly interpolated between the discrete data points.

count was increased by one read per million reads in the library (cpm increased by one). Enrichment level was determined in the same fashion, but using the full gene length (including introns) and adding 0.5 to the RPKM instead of to the cpm, to even out the background levels between the samples.

The average coverage over genes and promoters was determined from the $100 \mathrm{bp}$ bin counts. Each gene was assigned 150 bins, with bin size 1/50 of the gene width, covering the gene and one gene width on either side. These bins were assigned coverages by averaging the read counts of all overlapping $100 \mathrm{bp}$ bins. Bins in the same position of each gene were then averaged over genes in the same expression quartile. The average coverage in a bin was calculated as the mean over the bin and the two neighboring bins to make the plot smoother. Genes with extremely high RPKM (larger than 100) were excluded from the analysis, to allow the mean to be calculated on a linear scale without being dominated by a few outlying genes. In our dataset, all the removed genes are ribosomal or mitochondrial.

All code for the analysis can be found in the supplementary material. In addition a flow chart outlining the analysis steps is shown is Supplementary Figure 3.

\section{RESULTS}

Using an immunoprecipitation of Histone $\mathrm{H} 3$ as a background sample is attractive for accounting for uneven coverage across the genome due to both technical and biological artifacts. Specifically, an $\mathrm{H} 3$ pull-down not only mimics all the steps in the ChIP-seq processing but data also locates the possible regions of the genome that have the $\mathrm{H} 3$ protein and therefore the potential to harbor a histone modification. In order to assess the possible advantages in using an $\mathrm{H} 3$ control we began by comparing $\mathrm{H} 3$ with a standard WCE background sample.

\section{COMPARING BACKGROUND SAMPLES ACROSS THE GENOME}

As we have outlined, control samples are often used to cancel background reads that can lead to false signals in the histone modification samples. In order to verify that the controls indeed have structure beyond random sampling, such as enriched or depleted regions, we examined the distribution of reads across the genome. Initially we counted the number of reads in $1 \mathrm{~kb}$ bins across the genome and compared the count distributions between WCE, $\mathrm{H} 3$ and simulated random counts from a Poisson distribution. To account for different library sizes, the larger libraries were downsampled to match the smallest one (see Section Methods). The WCE and H3 count distributions are very similar to each other and they both display heavier tails compared with a Poisson distribution, thus implying non-random structures in the data (Figure 1). We see more bins with high counts than what is expected from a random Poisson distribution, which is an indication of enriched regions. The nearly identical distributions of the WCE and $\mathrm{H} 3$ samples in our data show that both control samples have similar number and size of enriched regions.

We next investigated whether these suggested non-random features coincide in location on the genome between the background samples. To do this we performed a statistical test for differential enrichment between the $\mathrm{H} 3$ and WCE counts in $1 \mathrm{~kb}$ bins using limma and voom (Smyth, 2004; Law et al., 2014), and plotted the fold changes in an MA plot (Figure 2A). In the limma-voom analysis the three H3K27me3 samples are included for improved estimation of the variance between samples. The most striking difference between the WCE and $\mathrm{H} 3$ samples occurs at mitochondrial DNA where there is enrichment prevalent in the WCE sample but none in the $\mathrm{H} 3$ samples. This is expected since mitochondrial DNA is not packaged by histones and therefore is not recognized by the H3 antibody (Chen and Butow, 2005). In addition, the ribosomal gene $R n 45 s$ shows exceptionally high counts in both data sets, but significantly higher counts in WCE than $\mathrm{H} 3$. This ribosomal gene is present in a large but varying number of copies on the genome (http://www.ncbi.nlm.nih.gov/ gene/100861531), and these regions are known to cause false positives in CHIP-seq data (Pickrell et al., 2011) The remainder of the significantly different bins are intergenic repeat regions, with large counts in both WCE and H3 but modest fold changes, and are marked by RepeatMasker (Kent et al., 2002; Tempel, 2012) as repetitive elements. It is important to note that apart from the enrichment in the mitochondrial DNA we again see that the two background samples have enrichment locations that are more similar to each other than they are to a randomly generated background sample (Supplementary Figure 1). In summary the vast majority of bin counts across the genome are very similar between the control samples.

\section{COMPARING BACKGROUND SAMPLES OVER GENES}

We next shifted our focus to the behavior of the background samples in and around genes, as these are often the regions of most interest in functional studies. Figure 2B shows an MA plot comparing the number of reads in each gene for WCE and H3. For this analysis significance is tested using the total number of reads across the entire gene, which for most genes gives more power than the $1 \mathrm{~kb}$ bin count. We find 444 genes are differentially 


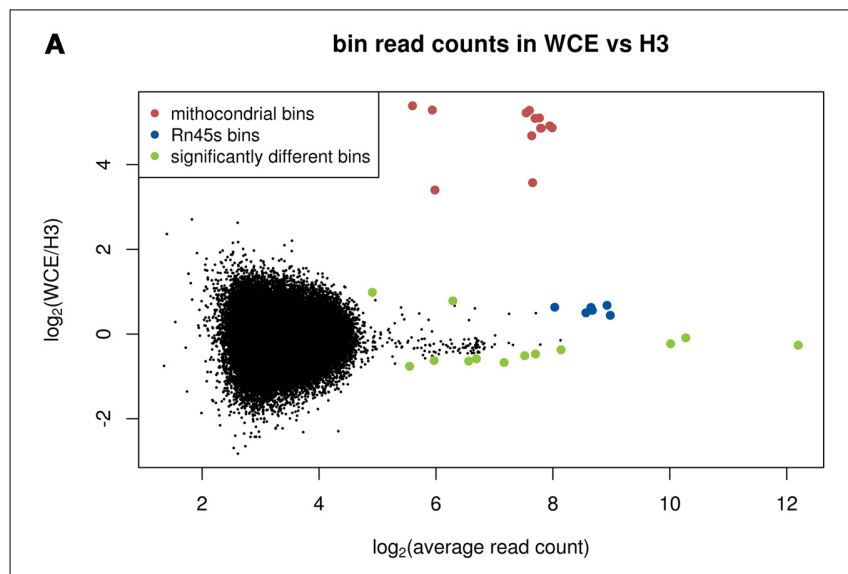

$\mathbf{B}$

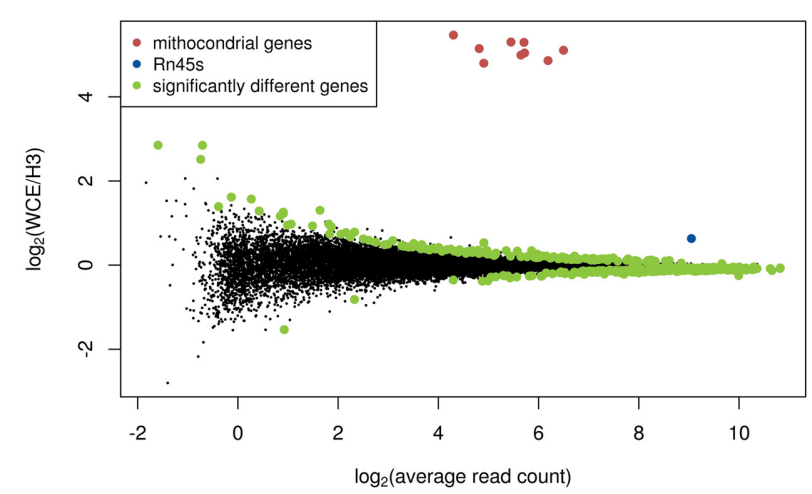

FIGURE 2 | MA plots comparing enrichment between WCE and H3: (A) $1 \mathbf{k b}$ bins across the genome, (B) bins that span genes. Significantly different bin counts between samples are highlighted and $R n 45 s$ and mitochondrial bins (all significantly different) are highlighted in blue and red, respectively. Significance is defined as FDR below 0.05 in a limma-voom analysis.

enriched with an FDR below 0.05. However, only 35 of the significantly different genes have a fold change larger than a factor 1.5. Consistent with the analysis of bin counts above, it is only the mitochondrial genes and $R n 45 s$ that stand out from the rest of the population as being more highly enriched in the WCE compare with the $\mathrm{H} 3$ sample.

\section{COMPARISONS OF DETECTED PEAKS}

The bin counts analysis above is not optimized to capture enrichment features that either span multiple bins or are significantly smaller than the bin size. Therefore, we next identified enriched regions in the data using MACS to call peaks in our background samples. MACS (Zhang et al., 2008) is designed to find enriched peaks in the coverage of ChIP-seq data, and scores the peaks according to significance.

MACS identified 856 peaks in the mouse WCE sample, and 867 peaks in the merged H3 samples, with 677 WCE peaks overlapping an $\mathrm{H} 3$ peak. We found that overlapping peaks between WCE and H3 were assigned similar scores by MACS (Figure 3B, Pearson correlation 0.91). We also noted that the peaks that appeared in one of the controls, but not in the other are scored much lower than the peaks that are common between the samples (Figure 3A). These two observations show that WCE and H3 have very similar peak locations.

We next performed a standard peak finding analysis of the merged H3K27me3 samples, using either WCE or H3 as a control. For WCE and H3, MACS returned 28,823 and 32,462 regions, respectively in the $\mathrm{H} 3 \mathrm{~K} 27 \mathrm{me} 3$ data. The vast majority of these peaks were overlapping $(26,113$ of the peaks with WCE as control overlap a peak with $\mathrm{H} 3$ as control). The enriched regions that are detected with both background samples have highly correlated peak scores (Figure 3D, Pearson correlation 0.97), and the few peaks that are unique to the analysis for only one of the background samples have very low scores (Figure 3C). This shows that the small differences that exist between our WCE and H3 samples have a negligible impact in a typical peak finding analysis and would not be prioritized for follow up.

Using any of the controls on our data set improved the quality of the analysis. Without the controls, only 9565 peaks were called in the $\mathrm{H} 3 \mathrm{~K} 27 \mathrm{me} 3$ samples, and many biologically relevant peaks were lost (Supplementary Methods and Supplementary Figure 2).

\section{COMPARISON OF H3K27me3 TO EXPRESSION WITH DIFFERENT BACKGROUND SAMPLES}

It is known that $\mathrm{H} 3 \mathrm{~K} 27 \mathrm{me} 3$ enrichment at the promoter or body of a gene is associated with low expression of that gene (Young et al., 2011). First we used expression levels estimated from our RNA-seq data to split the genes into four equal groups, or quartiles, based on expression from least to most expressed. We then plotted the average read density of genes in each quartile for each of our ChIP-seq and background samples (Figures 4A-F).

As expected, we found that $\mathrm{H} 3 \mathrm{~K} 27 \mathrm{me} 3$ is more enriched across gene bodies for lowly expressed genes, and has pronounced enrichment over the transcription start site for the lower expression quartiles. By contrast, both WCE and $\mathrm{H} 3$ have somewhat higher coverage in highly expressed genes. The WCE sample displays a peak over the transcription start site (TSS) for the highly expressed genes, whereas the $\mathrm{H} 3$ and $\mathrm{H} 3 \mathrm{~K} 27 \mathrm{me} 3$ samples display a dip or trough at the TSS. The dip for $\mathrm{H} 3$ and $\mathrm{H} 3 \mathrm{~K} 27 \mathrm{me} 3$ is consistent with a depletion of nucleosomes at the TSS (Ozsolak et al., 2007), which also could contribute to the peak in the WCE sample. We then divided the H3K27me3 coverage by the coverage of the controls, and found that the H3K27me3 dip was perfectly canceled out by the $\mathrm{H} 3$ control, while dividing by WCE reinforced the dip (Figures $4 \mathbf{G}, \mathbf{H}$ ). The perfect cancelation shows that the dips in $\mathrm{H} 3 \mathrm{~K} 27 \mathrm{me} 3$ and $\mathrm{H} 3$ likely are from the same source. This difference between $\mathrm{H} 3$ and WCE is important, as the depletion of histone modifications over the TSS compared to WCE might be misinterpreted as histones around the TSS being unmodified. Using the $\mathrm{H} 3$ as control shows that the dip in $\mathrm{H} 3 \mathrm{~K} 27 \mathrm{me} 3$ at the TSS is due to a lack of histones in general: the few histones that are there carry modifications as frequently as other histones close by. This difference between the controls is discussed further in the discussion.

One overall measure of quality of the ChIP-seq data is how well the $\mathrm{H} 3 \mathrm{~K} 27 \mathrm{me} 3$ histone modification anti-correlates with gene expression. To investigate this we used the number of reads (RPKM) in the gene body or the promoter (defined as $4 \mathrm{~kb}$ 
A

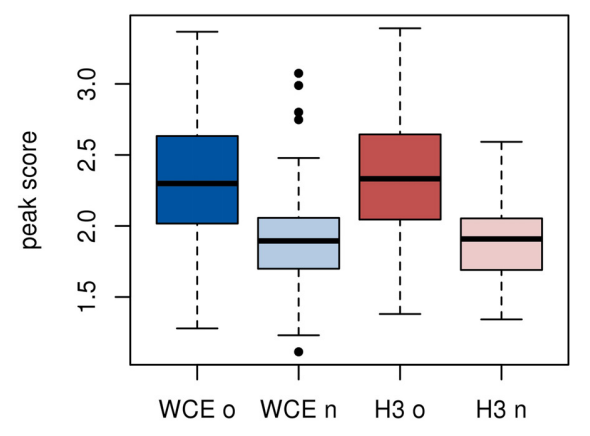

C

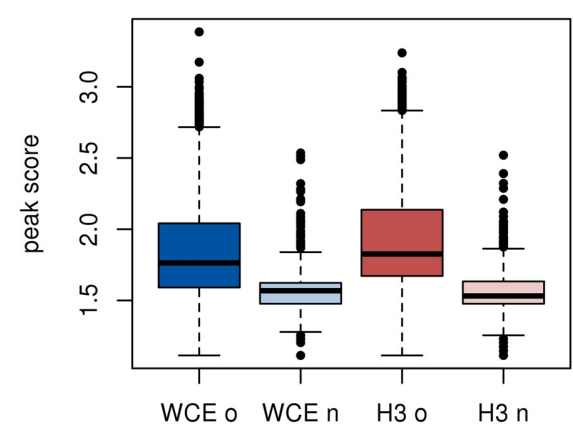

B

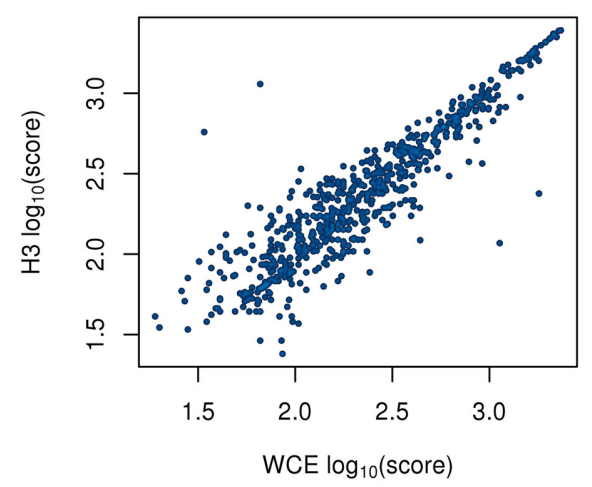

scores of overlapping peaks

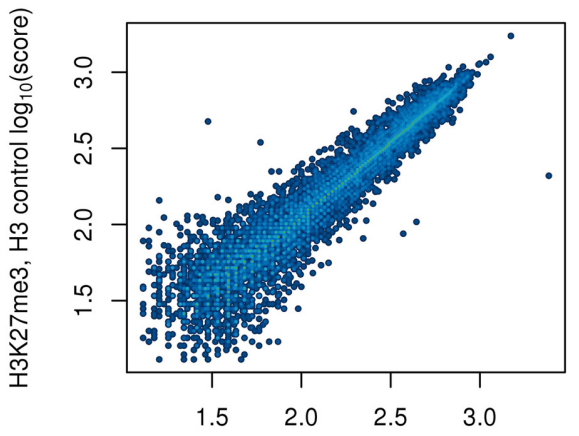

H3K27me3, WCE control $\log _{10}$ (score)
FIGURE 3 | Peak scores from a MACS analysis of enriched regions. $\mathrm{H} 3$ and H3K27me3 replicates are merged before analysis. (A) Distributions of scores of peaks in WCE and H3. The peaks in each control are split into peaks that overlap between the two background samples (marked with "o") or do not overlap (marked with " $n$ "). (B)
Scatter plot of scores of overlapping peaks from the WCE and H3 sample. (C) Distributions of scores of peaks in H3K27me3 with either WCE or $\mathrm{H} 3$ as a control. Labels on the x-axis refer to the control sample. (D) Scatter plot of scores of overlapping peaks from H3K27me3 with either WCE or $\mathrm{H} 3$ as control. centered at the TSS) for the H3K27me3 mark (Table 1). We then calculated the same correlation after dividing by the counts (RPKM) measured in the WCE or H3 control samples. Table 1 shows the Pearson correlations between the log of the ChIP-seq enrichment at genes (RPKM or ratio of RPKMs) and log of the expression (RPKM).

As expected, H3K27me3 enrichment over both the gene body and the promoter show an anti-correlation with expression. However, dividing by a control sample greatly improves the strength of the correlation for both the gene body and promoter. The WCE background improved the correlation slightly more than the $\mathrm{H} 3$ samples, especially for the promoter region. Both controls have a fairly strong positive correlation to expression, indicating that there likely is a positive background contribution to the H3K27me3 correlation, canceling out part of the negative correlation. This background contribution was removed by dividing by the control samples, and the full negative correlation from the histone modification appears.

To investigate this effect further, we took data from Adli et al. (2010) on H3K27me3 and H3K36me3 enrichment in adult mouse hematopoietic stem and progenitor cells, and calculated the correlations using our WCE and H3 as background samples. These datasets are from a cell type that is more similar to that used for our expression data, giving stronger correlations overall, however the same trends were observed in relation to the control samples. The H3K27me3 data behaved the same as in our dataset, in that both controls strengthened the anti-correlation between H3K27me3 enrichment and expression, and the WCE provided stronger anti-correlation than the $\mathrm{H} 3$ control. Conversely, the $\mathrm{H} 3 \mathrm{~K} 36 \mathrm{me} 3$ correlation to expression was strong without controls, but weakened by the introduction of controls. Again, the effect was strongest in the promoter with WCE as control. It should be noted here that the weaker correlation in the H3K36me3 at the promoter is expected, as $\mathrm{H} 3 \mathrm{~K} 36 \mathrm{me} 3$ is an elongation mark, and is normally enriched over exons rather than promoters. These results can be interpreted as both $\mathrm{H} 3 \mathrm{~K} 27 \mathrm{me} 3$ and $\mathrm{H} 3 \mathrm{~K} 36 \mathrm{me} 3$ having their correlation to expression influenced by the background. The control samples are both positively correlated to expression, indicating that the background likely increases the correlation for the histone modifications. This explains why the anti-correlation of expression with $\mathrm{H} 3 \mathrm{~K} 27 \mathrm{me} 3$ is strengthened by the controls, while for $\mathrm{H} 3 \mathrm{~K} 36 \mathrm{me} 3$ the correlation is weakened. 

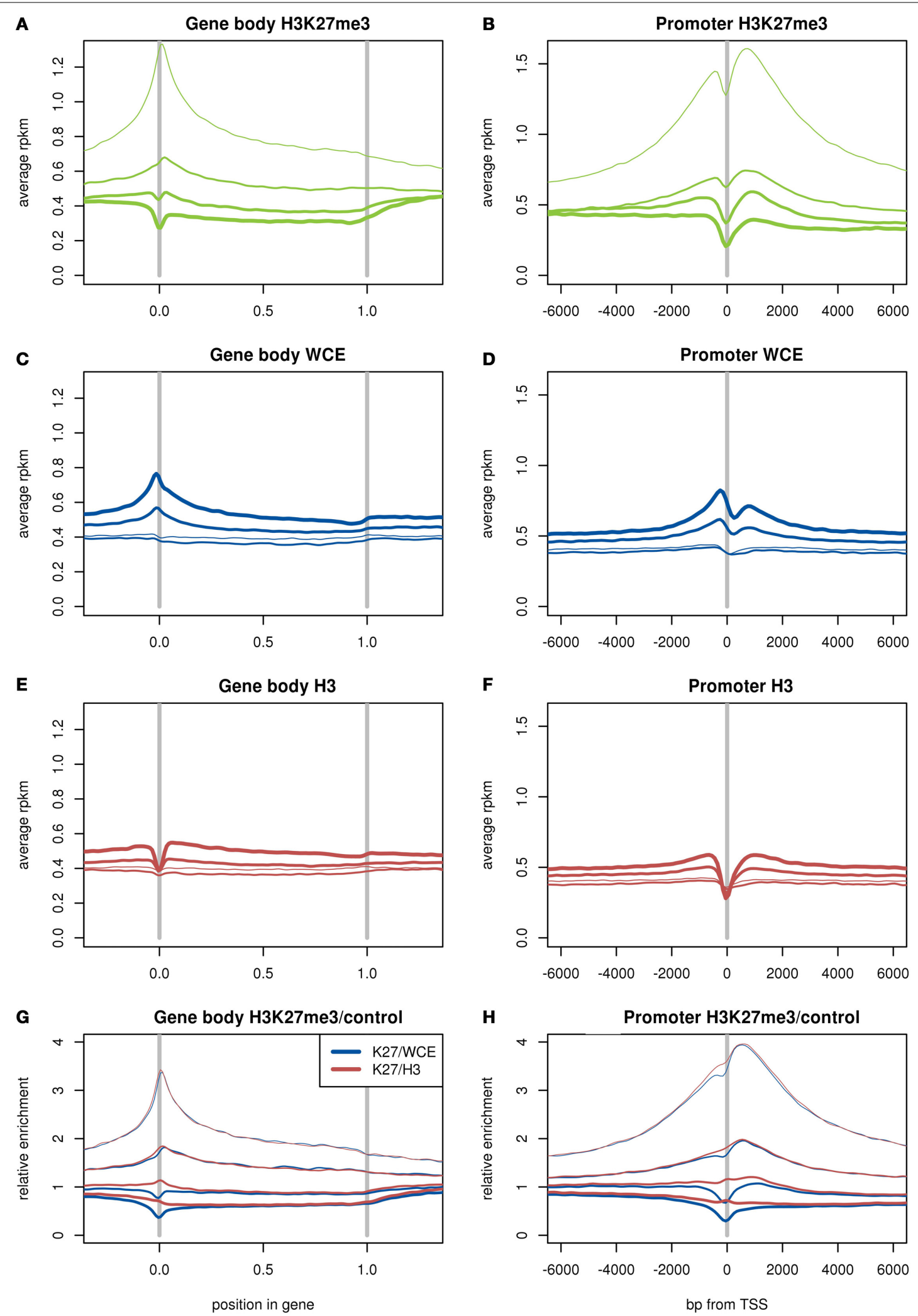

FIGURE 4 | Average read density in RPKM over genes (A,C,E) and around the transcription start site $(B, D, F)$ for H3K27me3 (A,B), WCE (C,D), and $\mathbf{H 3}(\mathbf{E}, \mathbf{F})$. Ratio of average read densities of H3K27me3 to the background sample over genes (G) and around the TSS (H). The

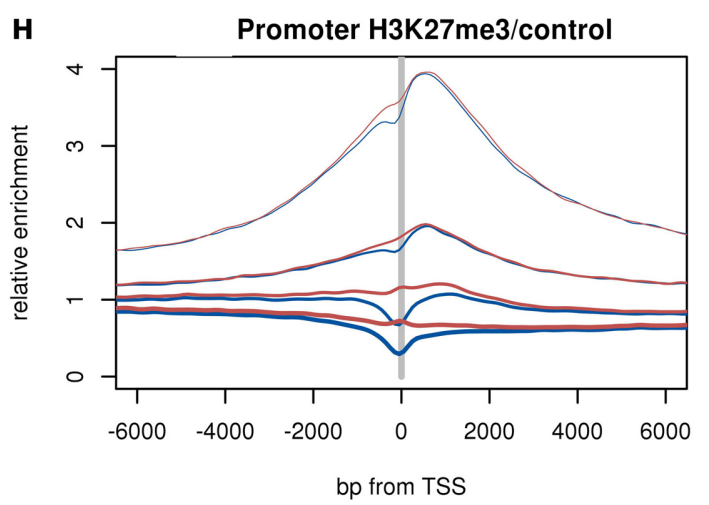

genes are divided up into four equal groups based on expression (RPKM), shown by thin lines for lowest expression and thicker lines for higher expression. The two H3 samples and three H3K27me3 samples are merged. 
Table 1 | Pearson correlation between expression and enrichment of our H3K27me3 data and H3K27me3 and H3K36me3 from Adli et al. (2010).

\begin{tabular}{lccccc}
\hline & WCE & H3 & H3K27me3 & H3K27me3/WCE & H3K27me3/H3 \\
\hline Gene body & 0.3 & 0.27 & -0.23 & -0.46 & -0.44 \\
Promoter & 0.35 & 0.25 & -0.26 & -0.57 & -0.51 \\
\hline Adli et al. & & H3K27me3 & H3K27me3/WCE & H3K27me3/H3 \\
\hline Gene body & & -0.51 & -0.64 & -0.63 \\
Promoter & & -0.49 & -0.66 & -0.63 \\
\hline Adli et al. & & H3K36me3 & H3K36me3/WCE & H3K36me3/H3 \\
\hline Gene body & & 0.69 & 0.59 & 0.62 \\
Promoter & & 0.43 & 0.26 & 0.35 \\
\hline
\end{tabular}

Enrichment is measured by the logarithm of RPKM, or logarithm of ratio of RPKM in the case of the modification with control samples, and expression is measured by the logarithm of RPKM of the RNA-seq samples.

\section{DISCUSSION}

We have generated ChIP-seq data for Histone H3, H3K27me3, and WCE samples from a hematopoietic stem and progenitor cell population isolated from E14.5 mouse fetal liver from C57BL/6 mice, and mRNA-seq expression data from a hematopoietic stem and progenitor cell population isolated from the bone marrow of adult mice that had received fetal liver cell transplants. We used these data, together with publicly available data, to compare WCE and $\mathrm{H} 3$ for their efficacy as control samples for modified histone ChIP-seq data.

Using WCE or H3 as a control yields slightly different interpretations of the corrected data. Dividing a histone modification ChIP-seq sample by a WCE background normalizes the sample against genome and fragmentation biases, with no explicit regard to the underlying histones. In contrast, dividing by a $\mathrm{H} 3$ background estimates the enrichment relative to the position of histones in the sample. Which of these two measures is more appropriate depends on the experiment, and the type of biology being probed. For example, in a study looking at the relationship between the H3K27me3 density and the compactness of the genome it may be more relevant to compare $\mathrm{H} 3 \mathrm{~K} 27$ me 3 density to a WCE control. On the other hand, using $\mathrm{H} 3$ as a background sample might be more relevant for a study looking at the how PRC2 affects the methylation of histones tails where we need to know which histones are modified. However, most experiments with ChIP-seq of histone modifications do not have the precision to tell the two interpretations apart, and we will focus on which control sample can extract the most information from the experiment.

We found, as has previously been observed, that both the WCE and H3 samples have more features and structures than would be expected from randomly distributed reads. For example, both controls are more enriched over highly expressed genes than over lowly expressed genes. We compared read counts in $1 \mathrm{~kb}$ bins between WCE and $\mathrm{H} 3$ and found that most features of the read distributions are similar between background samples. The notable exception to this was mitochondrial DNA where no $\mathrm{H} 3$ histones are present. The non-random enrichment features seen in both background samples can occur from biological sources, such as accessibility of the DNA, stability of the interaction between DNA fragments and targeted proteins as well as technical processes such as PCR artifacts and sequencing biases. Furthermore, biases can arise from the data analysis, with mappability perhaps being the most influential. These error sources are also expected to be present in ChIP-seq of histone modifications, and thus the WCE and $\mathrm{H} 3$ are suitable as background controls, with the majority of the structures in both WCE and H3 capable of canceling out biases.

When the data is averaged over a large number of genes, a more relevant difference between the control samples become apparent. WCE shows more enrichment over the TSS for expressed genes, while $\mathrm{H} 3$ and $\mathrm{H} 3 \mathrm{~K} 27 \mathrm{me} 3$ show a trough at the TSS. The trough seen in both the $\mathrm{H} 3$ and $\mathrm{H} 3 \mathrm{~K} 27 \mathrm{me} 3$ pull-down is likely due to nucleosome depletion specifically at the TSS where the transcriptional machinery is accessing the DNA (Ozsolak et al., 2007). Possibly the peak in WCE is due to the same reason, where the WCE extraction instead will collect a larger number of reads from regions that are depleted of nucleosomes. The small dip in WCE coverage just after the TSS could then be caused by the first nucleosome, often located in roughly the same position in expressed genes as was shown in Ozsolak et al. (2007). This difference between WCE controls and histone ChIP samples can have an impact on an analysis of expressed genes in the TSS region as can be seen in Figure $\mathbf{4 H}$.

Apart from very specific analyses averaged over many genes around the TSS, the two control samples give very similar results. Using WCE or $\mathrm{H} 3$ as the control for peak finding in $\mathrm{H} 3 \mathrm{~K} 27 \mathrm{me} 3$ ChIP-seq data returned essentially the same peaks (less than $1 \%$ of peaks were not concordant when called against WCE or H3), and the peak scores had a Pearson correlation of 0.996 . We believe that in the majority of cases the choice of which background sample is used will make a negligible difference to the results. Therefore, the use of WCE or input as a standard control sample is well justified.

\section{ACKNOWLEDGMENTS}

This work was supported by NHMRC grants (APP105140 to Alicia Oshlack and APP1027398 to Marnie E. Blewitt and Alicia Oshlack). Alicia Oshlack was supported by a Career Development Fellowship from the NHMRC. Marnie E. Blewitt was supported by an Australian Research Council, Queen Elizabeth II Fellowship. This work was made possible through Victorian State Government Operational Infrastructure Support and Australian National Health and Medical Research Council Research Institute Infrastructure Support Scheme.

\section{SUPPLEMENTARY MATERIAL}

The Supplementary Material for this article can be found online at: http://www.frontiersin.org/journal/10.3389/fgene. 2014.00329/abstract

\section{REFERENCES}

Adli, M., Zhu, J., and Bernstein, B. E. (2010). Genome-wide chromatin maps derived from limited numbers of hematopoietic progenitors. Nat. Methods 7, 615-618. doi: 10.1038/nmeth.1478 
Bernstein, B. E., Stamatoyannopoulos, J. A., Costello, J. F., Ren, B., Milosavljevic, A., Meissner, A., et al. (2010). The NIH roadmap epigenomics mapping consortium. Nat. Biotechnol. 28, 1045-1048. doi: 10.1038/nbt1010-1045

Bock, I., Dhayalan, A., Kudithipudi, S., Brandt, O., Rathert, P., and Jeltsch, A. (2011). Detailed specificity analysis of antibodies binding to modified histone tails with peptide arrays. Epigenetics 6, 256-263. doi: 10.4161/epi.6.2. 13837

Chen, X. J., and Butow, R. A. (2005). The organization and inheritance of the mitochondrial genome. Nat. Rev. Genet. 6, 815-825. doi: 10.1038/ nrg 1708

Consortium, Encode Project. (2004). The ENCODE (ENCyclopedia Of DNA Elements) Project. Science 306, 636-640. doi: 10.1126/science.1105136

Ho, J. W., Bishop, E., Karchenko, P. V., Negre, N., White, K. P., and Park, P. J. (2011). ChIP-chip versus ChIP-seq: lessons for experimental design and data analysis. BMC Genomics 12:134. doi: 10.1186/1471-2164-12-134

Kent, W. J., Sugnet, C. W., Furey, T. S., Roskin, K. M., Pringle, T. H., Zahler, A. M., et al. (2002). The human genome browser at UCSC. Genome Res. 12, 996-1006. doi: 10.1101/gr.229102

Kidder, B. L., Hu, G., and Zhao, K. (2011). ChIP-Seq: technical considerations for obtaining high-quality data. Nat. Immunol. 12, 918-922. doi: 10.1038/ni.2117

Koboldt, D. C., Steinberg, K. M., Larson, D. E., Wilson, R. K., and Mardis, E. R. (2013). The next-generation sequencing revolution and its impact on genomics. Cell 155, 27-38. doi: 10.1016/j.cell.2013.09.006

Landt, S. G., Marinov, G. K., Kundaje, A., Kheradpour, P., Pauli, F., Batzoglou, S., et al. (2012). ChIP-seq guidelines and practices of the ENCODE and modENCODE consortia. Genome Res. 22, 1813-1831. doi: 10.1101/gr.136184.111

Law, C. W., Chen, Y., Shi, W., and Smyth, G. K. (2014). Voom: precision weights unlock linear model analysis tools for RNA-seq read counts. Genome Biol. 15:R29. doi: 10.1186/gb-2014-15-2-r29

Liang, K., and Keles, S. (2012). Normalization of ChIP-seq data with control. BMC Bioinformatics 13:199. doi: 10.1186/1471-2105-13-199

Ozsolak, F., Song, J. S., Liu, X. S., and Fisher, D. E. (2007). High-throughput mapping of the chromatin structure of human promoters. Nat. Biotechnol. 25, 244-248. doi: 10.1038/nbt1279

Park, P. J. (2009). ChIP-seq: advantages and challenges of a maturing technology. Nat. Rev. Genet. 10, 669-680. doi: 10.1038/nrg2641

Pickrell, J. K., Gaffney, D. J., Gilad, Y., and Pritchard, J. K. (2011). False positive peaks in ChIP-seq and other sequencing-based functional assays caused by unannotated high copy number regions. Bioinformatics 27, 2144-2146. doi: 10.1093/bioinformatics/btr354

Smyth, G. K. (2004). Linear models and empirical bayes methods for assessing differential expression in microarray experiments. Stat. Appl. Genet. Mol. Biol. 3:3. doi: 10.2202/1544-6115.1027

Tempel, S. (2012). Using and understanding RepeatMasker. Methods Mol. Biol. 859, 29-51. doi: 10.1007/978-1-61779-603-6_2

Trynka, G., Sandor, C., Han, B., Xu, H., Stranger, B. E., Liu, X. S., et al. (2013). Chromatin marks identify critical cell types for fine mapping complex trait variants. Nat. Genet. 45, 124-130. doi: 10.1038/ng.2504

Young, M. D., Willson, T. A., Wakefield, M. J., Trounson, E., Hilton, D. J., Blewitt, M. E., et al. (2011). ChIP-seq analysis reveals distinct H3K27me3 profiles that correlate with transcriptional activity. Nucleic Acids Res. 39, 7415-7427. doi: 10.1093/nar/gkr416

Zhang, Y., Liu, T., Meyer, C. A., Eeckhoute, J., Johnson, D. S., Bernstein, B. E., et al. (2008). Model-based analysis of ChIP-Seq (MACS). Genome Biol. 9:R137. doi: 10.1186/gb-2008-9-9-r137

Zhu, J., Adli, M., Zou, J. Y., Verstappen, G., Coyne, M., Zhang, X., et al. (2013). Genome-wide chromatin state transitions associated with developmental and environmental cues. Cell 152, 642-654. doi: 10.1016/j.cell.2012.12.033

Conflict of Interest Statement: The authors declare that the research was conducted in the absence of any commercial or financial relationships that could be construed as a potential conflict of interest.

Received: 15 July 2014; accepted: 03 September 2014; published online: 25 September 2014.

Citation: Flensburg C, Kinkel SA, Keniry A, Blewitt ME and Oshlack A (2014) A comparison of control samples for ChIP-seq of histone modifications. Front. Genet. 5:329. doi: 10.3389/fgene.2014.00329

This article was submitted to Bioinformatics and Computational Biology, a section of the journal Frontiers in Genetics.

Copyright (c) 2014 Flensburg, Kinkel, Keniry, Blewitt and Oshlack. This is an openaccess article distributed under the terms of the Creative Commons Attribution License (CC BY). The use, distribution or reproduction in other forums is permitted, provided the original author(s) or licensor are credited and that the original publication in this journal is cited, in accordance with accepted academic practice. No use, distribution or reproduction is permitted which does not comply with these terms. 